\title{
Pathway-Supporting Health Information Systems: A Review
}

\author{
Tim SCHEPLITZ ${ }^{\mathrm{a}, 1}$ \\ ${ }^{a}$ Technische Universität Dresden, Dresden, Germany
}

\begin{abstract}
Care pathways and supporting health information systems (HIS) have been permeate the discipline of Health Information Systems Research (HISR) over years. Traditional objectives of workflow assistance are increasingly extended by interdisciplinary goals from technology, medicine, management and public health research. A systematic literature review is dedicated to this integrating character. It examines the interdisciplinary mesh of objectives associated with care pathways and pathway-supporting HIS in the HISR literature. From 47 identified articles, 6 thematic themes were derived. Their consolidation supports in particular design and development processes as it describes the solution space of future pathwaysupporting HIS addressing requirements stated by multiple stakeholders.
\end{abstract}

Keywords. Care pathways, health information system, pathway systems, review

\section{Introduction}

In medical practice and research care pathways describe complex sequences of interventions of defined patient groups in defined time periods to support the organization, coordination and decision making of care processes [1]. Different terms are used to emphasize intra-organizational ("clinical pathways"), inter-institutional ("integrated pathways") or patient-centered ("patient pathways") orientations [2]-[4]. Also Health Information Systems Research (HISR) investigates care pathways especially the conception, modeling, realization, and impact of pathway-supporting Health Information Systems (HIS) [5]-[7].

In this context, the research and development work seek to bring original motivations from the process perspective in line with the requirements of innovative disciplines. For example, it is being discussed how patient integration can be intensified along care pathways [7], how data mining methods can describe care pathways retrospectively [8], or how data analytics approaches can contribute to the individualization of care plans [9]. Experiences from practice-oriented digital health projects underline the observation that the objectives of care pathways and pathwaysupporting HIS go beyond the traditional process support.

This paper follows up on these observations. If research and development teams want to master the multidisciplinary field of requirements for pathways-supporting HIS, a consolidation of previous work can support them especially in conceptual design and development process. Such a compilation can describe the solution space for pathways-

Corresponding Author: Tim Scheplitz, Technische Universität Dresden, Germany, tim.scheplitz@tu-dresden.de 
supporting HIS, derive design implications, and point to disciplines and professions to be involved. This review paper therefore addresses the question of how the literature discusses scope and challenges of pathway-supporting HIS and which contributions can be used to face the mentioned mission of multidisciplinary requirements engineering. The review thus extends the knowledge from previous reviews on characterization of patient pathways [2] and on support opportunities of clinical pathways by Health Information Technologies [10].

\section{Methods}

A systematic literature review [11]-[13] for the mentioned research question was conducted in the last quarter of 2020. The following high-quality databases of ISR or HISR were chosen: AIS Senior Basket, Proceedings of the AIS Conferences, recommended eHealth Journals of the AIS SIG Health. The past decade was chosen as the publication period. After abstract and full-text screening, 47 articles were defined as final set for analysis. Only articles that prominently named care pathways as a research context were included. Review articles were not excluded from the analysis set in order to extract implications of their contributions to research and practice. Further details of the search process are given in Figure.1.

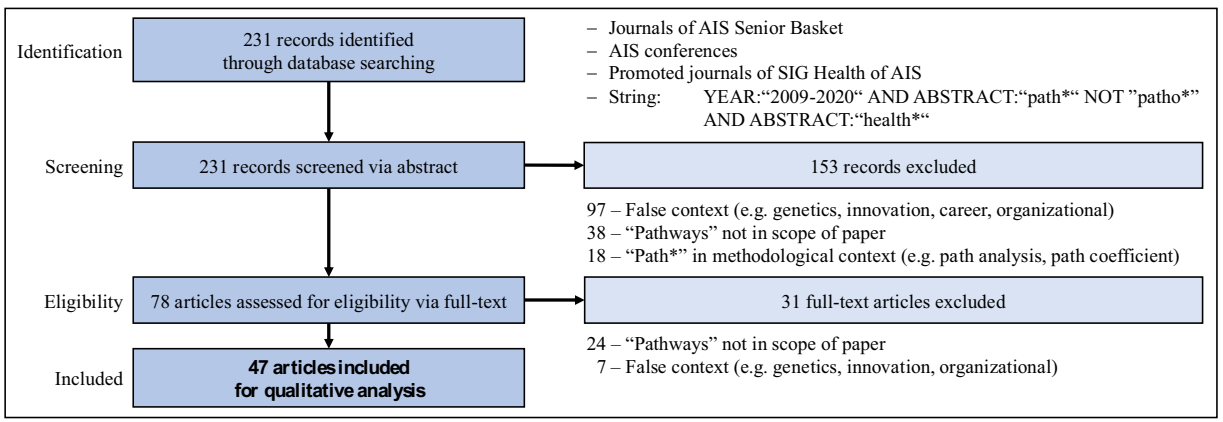

Figure 1. Review process according to PRISMA guidelines [13]

Following the process model of a summarizing content analysis with inductive category formation [14], the analysis material was reduced to those passages in which central goals or contributions related to care pathways or pathway-supporting HIS are described. Paraphrases were constructed and structured interpretatively. Through this structuring step, six themes were identified and validated in group session with three digital health experienced researchers.

\section{Results}

The 47 identified articles are distributed relatively evenly over the past years. With regard to the distribution across journals or conferences, it can be noted that the majority of the articles found are published in HISR journals recommended by the AIS Health SIG $(n=35)$. Here, 13 articles could be found in the International Journal of Medical Informatics and 11 papers in the journal BMC Medical Informatics and Decision Making. 
A total of 11 papers were identified in the proceedings of the AIS conferences, and only one article in the AIS Senior Basket.

Table 1 presents exemplary the results of paraphrasing step of the six articles published in 2020. It primarily shows how paraphrases have been formulated and offers secondly an insight about newest research on or with pathway-supporting HIS. A complete list of the paraphrasing results of all identified articles will be provided in additional material to this paper.

Table 1. Paraphrases examples of article analysis (only articles published in 2020)

Article [reference]: Summary of contribution's intersection to pathway-supporting HIS

Askari et al. 2020 [15]: Study for professional assessment of the effectiveness and efficiency of clinical pathways and path-supporting HIS in medical care

Cho et al. 2020 [16]: Development of a data-driven method for deriving clinical pathways from electronic health records

Gaveikaite et al. 2020 [17]: Case study along a COPD pathway for potential analysis of telemedicine

Kempa-Liehr et al. 2020 [18]: Modeling tool for clinical pathways and prediction models based on data mining methods and machine learning models for individual improvement of recovery time

Trajano et al. 2020 [19]: Process oriented modeling language for clinical pathways

Ye et al. 2020 [20]: Feasibility study on the use of deep learning prediction models for decision support and derivation of optimal, individual hypertension pathways based on electronic health records

Six themes have been inductively identified out of the paraphrasing results of all 47 articles. Some articles refer to up to two different themes due to their complexity (e.g. [18]). Table 2 shows all themes and sub-topics to give a referenced overview of pathwayrelated HISR of the last decade. The following additions highlight selected findings or implications for future design and implementation activities of pathway-supporting HIS.

I. Design, development and implementation of pathway-supporting HIS: This theme includes generic approaches as well as descriptions of pathway-supporting HIS and application systems for specific care scenarios. Such work has been established for years. The traditional workflow support of clinical processes is increasingly complemented by articles that present solutions for more complex, cross-institutional and patient-integrating care scenarios. Future work of this theme should concisely characterize the care scenario and parameterize targeted improvements to care-related goals or further outcomes in order to increase reusability.

II. Evaluation and assessment of pathway-supporting HIS: Theme II papers investigate the effects of pathway-supporting HIS. Increasingly complex care scenarios require future evaluative papers to consider all involved stakeholders more comprehensive (e.g. multidisciplinary care teams, patients, expanded health care market) and to discuss competing or synergistic effects and measurable outcomes.

III. Modeling and modeling languages of care pathways: Research with a focus on pathway modeling, modeling languages and tools discuss less the design and realization of application systems but address the underlying pathway models and their genesis. The established knowledge base provides already valuable guidance. However, the changing scope to integrated care scenarios with by multiple professional stakeholders and increasing technization may require additional work in this theme to offer appropriate techniques for precise, comprehensive and consistent pathway models.

IV. Data-driven pathway models and integration of data-based prediction models: Recent advances in data science, machine learning, and related disciplines drive articles in this young theme (earliest publication of this review from 2017). It includes articles that discuss the data-based derivation of care pathways from existing data, e.g. 
of EHR. Such retrospective pathway analyses provide the opportunity to compare originally defined care plans with de facto care pathways and to investigate deviations. In consequence, those knowledge might be used to optimize individual care pathways or general pathway templates prospectively or to improve medical guidelines (e.g. for care quality or efficiency). On the other hand, this theme also includes discussions of how pathway-supporting HIS can provide the data basis for data-driven medical decision support systems or management-oriented predictive models (linkage to Theme V). Both sub-themes - pathway-supporting HIS as a source and as a sink of data-driven processing of health information - like to merge and are currently of increasing interest.

V. Conceptual integration of the management perspective: Traditional workflow assistance fosters goals directly related to care processes such as accelerated process flow or lower error rates. Articles of this fifth theme investigate how pathway-supporting HIS can support additional short-, medium- and long-term tasks of healthcare management on micro, meso or macro level (e.g. quality management, resource management, health program management). Future work shall explore how pathway-supporting HIS need to be designed to satisfy this information demand and to offer management decision support. It therefore will probably benefit of popular research efforts of Theme IV.

VI. Care pathways as a means for HISR: Pathway-supporting HIS can be a starting point and data source for diverse research questions. Future design and implementation activities should consider the access to and scientific usability of the processed data and integrate validation and anonymization mechanisms in particular.

Table 2. Analytical results -themes on pathway-supporting HIS

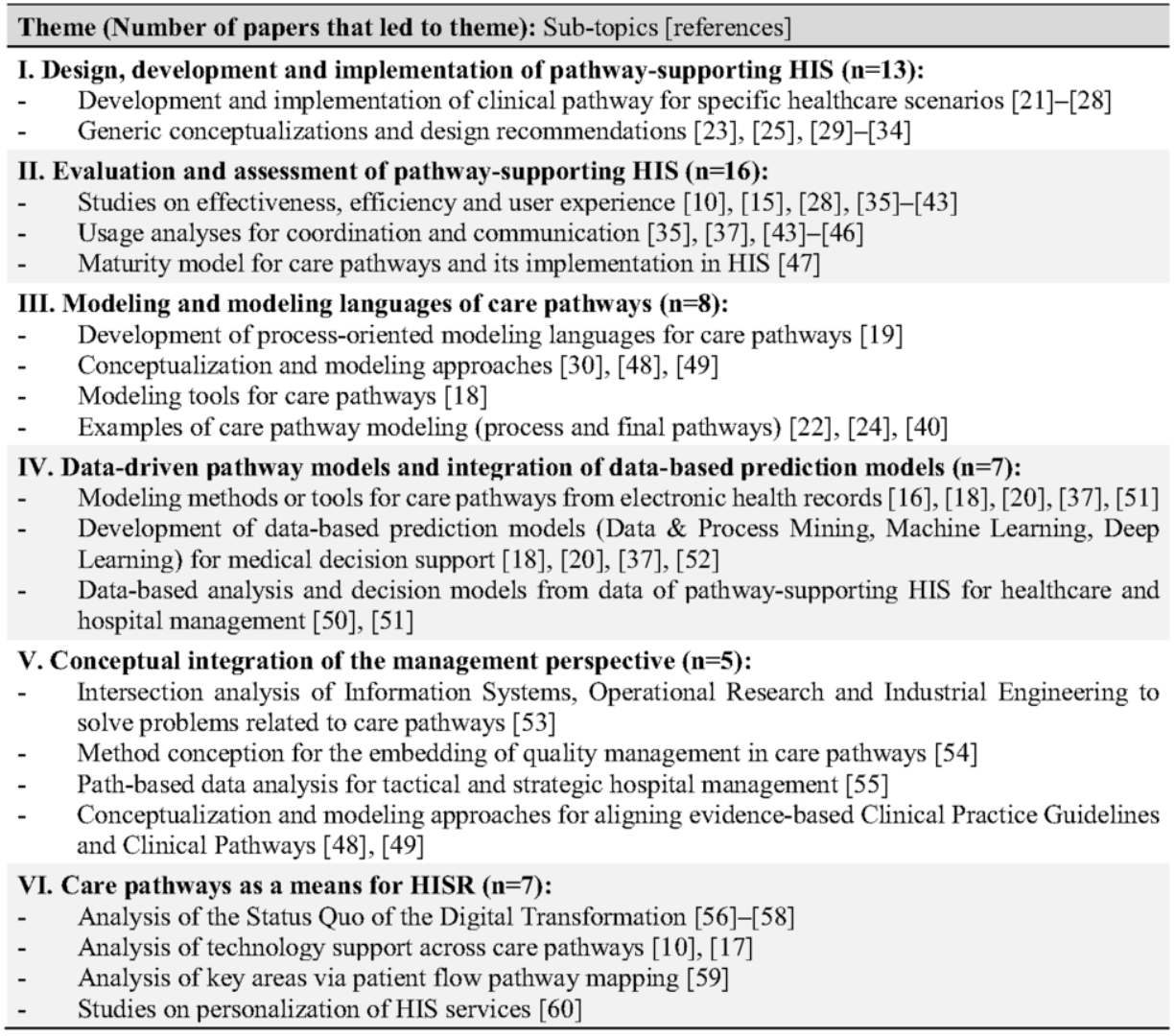




\section{Discussion}

The review results can be used for different tasks by research and development consortia of pathway-supporting HIS and offers an aid for their objectives. Institutions with a need to catch up in IT-based workflow support can draw on the contributions of Themes I and II to design and implement their own pathway-supporting HIS. The traditional care process support may seem almost obsolete compared to current HISR contributions. However, these themes remain particularly relevant to healthcare actors with low digital maturity. Contributions from Theme III should also be considered in this outlined case to enable the creation of the necessary pathway models as a success-critical resource. Theme III can also support consortia with already established pathway-supporting HIS and contribute to improvements of the used pathway models.

From the perspective of HISR and healthcare organizations of high digital maturity, Themes $I V$ and $V$ are highlighted in particular. Technological advances in data science and related disciplines indicate enhanced potential for both personalized care and learning systems of a macro level for management and medicine. Future work may generate data-based individual improvements in care pathways (e.g., based on patient constitution or resource availability) or address care economic issues at the local (e.g., clinical process improvements) or global level (e.g., regionally adequate care programs). Future contributions will help pathway-supporting HIS to benefit from the technological progress while providing the required valid and consistent data for these mechanisms.

The contribution of this paper is affected by a certain number of limitations. For example, the analysis sample from the selected publication organs offers a solid basis for interpretation, but is still restricted. The integration of additional development-focused journals or conferences could provide more differentiation in the design of novel pathway-supporting HIS. Further, the creation of the six research themes is also subject to the limited objectivity of the author, although this influence was counteracted by group sessions with experienced digital health researchers.

How may future research stimulate the progress? With the assumption that concrete care scenarios will continue to be main drivers for technological innovation with and for pathway-support HIS, the relationship between care context and system design could be explored in more detail. Such investigations could determine those care scenarios that motivate the development of pathway-supporting HIS in particular or those which benefit only from dedicated functionalities. Therefore, an appropriate description model is required to define characteristics as well as types of care scenarios, which in turn could be linked to concrete care goals on micro or macro level. Those studies should focus on: care setting (outpatient, inpatient, rehabilitation); medical discipline (e.g., oncology, emergency medicine); indications (e.g., COPD, depression); patient's role (autonomous vs. paternalistic); degree of multi-professionality or number of institutions involved.

\section{Conclusion}

This review identified 47 articles from highly ranked literature on care pathways or pathway-supporting HIS. Through their interpretation, six themes were derived that represent the range of interdisciplinary goals with and for pathway-supporting HIS. Their consolidation supports future design and development processes by describing the solution space for pathway-supporting HIS. In particular, the design of new systems can benefit from this summary and interdisciplinary requirements management of multiple 
stakeholders can be supported. Among those themes, articles with a scope on data-driven pathway models, the integration of data-based healthcare prediction models as well as the enhancement to pathway-supporting HIS for management and operations currently represent exciting fields of activity for HISR and seeks to future work.

\section{References}

[1] EPA, "Care Pathways," Eurpean Pathway Assosciation: Care Pathways, 2019. http://e-p-a.org/carepathways/.

[2] P. Richter and H. Schlieter, "Understanding Patient Pathways in the Context of Integrated Health Care Services-Implications from a Scoping Review," presented at the Wirtschaftsinformatik 2019, Siegen, Feb. 2019.

[3] L. Kinsman, T. Rotter, E. James, P. Snow, and J. Willis, "What is a clinical pathway? Development of a definition to inform the debate," BMC Med, vol. 8, pp. 31-33, 2010.

[4] K. Vanhaecht, M. Panella, R. van Zelm, and W. Sermeus, "An overview on the history and concept of care pathways as complex interventions," International Journal of Care Pathways, vol. 14, no. 3, pp. 117-123, Sep. 2010, doi: 10.1258/jicp.2010.010019.

[5] H. Raphael, T. Lux, and V. Martin, "State-of-the-art prozessorientierter Krankenhausinformationssysteme.," in Wirtschaftsinformatik (2), 2009, pp. 689-698.

[6] M. Burwitz, H. Schlieter, and W. Esswein, "Modeling Clinical Pathways - Design and Application of a Domain-Specific Modeling Language,” Wirtschaftsinformatik Proceedings 2013, Jan. 2013, [Online]. Available: http://aisel.aisnet.org/wi2013/83.

[7] M. Benedict et al., "Patientenintegration durch Pfadsysteme," presented at the Wirtschaftsinformatik (WI 2019), Siegen, 2019.

[8] E. S. Prokofyeva, R. D. Zaytsev, and S. V. Maltseva, "Application of Modern Data Analysis Methods to Cluster the Clinical Pathways in Urban Medical Facilities," in 2019 IEEE 21 st Conference on Business Informatics (CBI), Moscow, Russia, Jul. 2019, pp. 75-83, doi: 10.1109/CBI.2019.00016.

[9] H. Schlieter, M. Benedict, K. Gand, and M. Burwitz, "Towards Adaptive Pathways: Reference Architecture for Personalized Dynamic Pathways,” Jul. 2017, pp. 359-368, doi: 10.1109/CBI.2017.55.

[10] M. T. Neame, J. Chacko, A. E. Surace, I. P. Sinha, and D. B. Hawcutt, "A systematic review of the effects of implementing clinical pathways supported by health information technologies," Journal of the American Medical Informatics Association, vol. 26, no. 4, pp. 356-363, Apr. 2019, doi: 10.1093/jamia/ocy176.

[11] G. Schryen et al., "Literature Reviews in IS Research: What Can Be Learnt from the Past and Other Fields?," Communications of the Association for Information Systems, vol. 41, no. 1, Dec. 2017, doi: 10.17705/1CAIS.04130.

[12] G. Paré, M.-C. Trudel, M. Jaana, and S. Kitsiou, "Synthesizing information systems knowledge: A typology of literature reviews," Information \& Management, vol. 52, no. 2, pp. 183-199, Mar. 2015, doi: 10.1016/j.im.2014.08.008.

[13] D. Moher, A. Liberati, J. Tetzlaff, D. G. Altman, and PRISMA Group, "Preferred Reporting Items for Systematic Reviews and Meta-Analyses: The PRISMA Statement," Ann Intern Med, vol. 151, no. 4, p. 264, Aug. 2009, doi: 10.7326/0003-4819-151-4-200908180-00135.

[14] P. Mayring, Qualitative Inhaltsanalyse: Grundlagen und Techniken, 12., Überarbeitete Auflage. Weinheim Basel: Beltz, 2015.

[15] M. Askari, J. L. Y. Y. Tam, M. F. Aarnoutse, and M. Meulendijk, "Perceived effectiveness of clinical pathway software: A before-after study in the Netherlands," International Journal of Medical Informatics, vol. 135, p. 104052, 2020, doi: https://doi.org/10.1016/j.ijmedinf.2019.104052.

[16] M. Cho et al., "Developing data-driven clinical pathways using electronic health records: The cases of total laparoscopic hysterectomy and rotator cuff tears," International Journal of Medical Informatics, vol. 133, p. 104015, Jan. 2020, doi: 10.1016/j.ijmedinf.2019.104015.

[17] V. Gaveikaite et al., "Challenges and opportunities for telehealth in the management of chronic obstructive pulmonary disease: a qualitative case study in Greece," BMC Med Inform Decis Mak, vol. 20 , no. 1, p. 216, Dec. 2020, doi: 10.1186/s12911-020-01221-y.

[18] A. W. Kempa-Liehr et al., "Healthcare pathway discovery and probabilistic machine learning," International Journal of Medical Informatics, vol. 137, p. 104087, 2020, doi: https://doi.org/10.1016/j.ijmedinf.2020.104087. 
[19] I. A. Trajano, J. B. F. Filho, F. R. de C. Souza, I. Litchfield, and P. Weber, "MedPath: A process-based modeling language for designing care pathways," International Journal of Medical Informatics, p. 104328, 2020, doi: https://doi.org/10.1016/j.ijmedinf.2020.104328.

[20] X. Ye, Q. T. Zeng, J. C. Facelli, D. I. Brixner, M. Conway, and B. E. Bray, "Predicting Optimal Hypertension Treatment Pathways Using Recurrent Neural Networks," International Journal of Medical Informatics, vol. 139, p. 104122, 2020, doi: https://doi.org/10.1016/j.ijmedinf.2020.104122.

[21] M. Donald et al., "Development and implementation of an online clinical pathway for adult chronic kidney disease in primary care: a mixed methods study.," BMC Medical Informatics \& Decision Making, vol. 16, pp. 1-11, 2016.

[22] R. S. Evans et al., "Automated identification and predictive tools to help identify high-risk heart failure patients: pilot evaluation," Journal of the American Medical Informatics Association, vol. 23, no. 5, pp. 872-878, Sep. 2016, doi: 10.1093/jamia/ocv197.

[23] V. Gkatzidou et al., "User interface design for mobile-based sexual health interventions for young people: Design recommendations from a qualitative study on an online Chlamydia clinical care pathway.," BMC Medical Informatics \& Decision Making, vol. 15, no. 1, pp. 1-13, 2015.

[24] S. Wagner et al., "Analysis and classification of oncology activities on the way to workflow based single source documentation in clinical information systems.," BMC Medical Informatics \& Decision Making, vol. 15 , pp. 1-13, 2015.

[25] M.-M. Bouamrane and F. S. Mair, "Integrated Preoperative Care Pathway - A Study of a Regional Electronic Implementation.," BMC Medical Informatics \& Decision Making, vol. 14, no. 1, pp. 1-32, 2014.

[26] J. E. Hurwitz, J. A. Lee, K. K. Lopiano, S. A. McKinley, J. Keesling, and J. A. Tyndall, “A flexible simulation platform to quantify and manage emergency department crowding.," BMC Medical Informatics \& Decision Making, vol. 14, no. 1, pp. 1-20, 2014.

[27] A. T.-H. Hao et al., "Nursing process decision support system for urology ward," International Journal of Medical Informatics, vol. 82, no. 7, pp. 604-612, 2013, doi: https://doi.org/10.1016/j.ijmedinf.2013.02.006.

[28] A. M. Ryhänen, S. Rankinen, K. Tulus, H. Korvenranta, and H. Leino-Kilpi, "Internet based patient pathway as an educational tool for breast cancer patients," International Journal of Medical Informatics, vol. 81, no. 4, pp. 270-278, 2012, doi: https://doi.org/10.1016/j.ijmedinf.2012.01.010.

[29] J. Gibbs et al., "The eClinical Care Pathway Framework: a novel structure for creation of online complex clinical care pathways and its application in the management of sexually transmitted infections.," BMC Medical Informatics \& Decision Making, vol. 16, pp. 1-9, 2016.

[30] W. Li, K. Liu, H. Yang, and C. Yu, "Integrated clinical pathway management for medical quality improvement - based on a semiotically inspired systems architecture," European Journal of Information Systems, vol. 23, no. 4, pp. 400-417, Jul. 2014, doi: 10.1057/ejis.2013.9.

[31] T. Paulussen, A. Heinzl, and C. Becker, "Multi-Agent Based Information Systems for Patient Coordination in Hospitals," ICIS 2013 Proceedings, 2013, [Online]. Available: https://aisel.aisnet.org/icis2013/proceedings/HealthcareIS/2/.

[32] K. Gand and H. Schlieter, "Personalisation and Dynamisation of Care pathways-Foundations and Conceptual Considerations.," in Proceedings of ECIS 2016, 2016, p. Research-in.

[33] S. Wakamiya and K. Yamauchi, "What are the standard functions of electronic clinical pathways?," International Journal of Medical Informatics, vol. 78, no. 8, pp. 543-550, 2009, doi: https://doi.org/10.1016/j.ijmedinf.2009.03.003.

[34] P. Gooch and A. Roudsari, "Computerization of workflows, guidelines, and care pathways: a review of implementation challenges for process-oriented health information systems," Journal of the American Medical Informatics Association, vol. 18, no. 6, pp. 738-748, Nov. 2011, doi: 10.1136/amiajnl-2010000033.

[35] M. Andellini et al., "Experimental application of Business Process Management technology to manage clinical pathways: a pediatric kidney transplantation follow up case.," BMC Medical Informatics \& Decision Making, vol. 17, pp. 1-9, 2017.

[36] A. Appari, M. E. Johnson, and D. L. Anthony, "Health IT and inappropriate utilization of outpatient imaging: A cross-sectional study of U.S. hospitals," International Journal of Medical Informatics, vol. 109, pp. 87-95, 2018, doi: https://doi.org/10.1016/j.ijmedinf.2017.10.020.

[37] L. Shivers, S. S. Feldman, and L. W. Hayes, "Development of a computerized paediatric intensive care unit septic shock pathway: improving user experience," Health Systems, pp. 1-7, May 2019, doi: 10.1080/20476965.2019.1620638.

[38] J. M. Toy, A. Drechsler, and R. C. Waters, "Clinical pathways for primary care: current use, interest and perceived usability," Journal of the American Medical Informatics Association, vol. 25, no. 7, pp. 901906, Jul. 2018, doi: 10.1093/jamia/ocy010. 
[39] J. Schuld, T. Schäfer, S. Nickel, P. Jacob, M. K. Schilling, and S. Richter, "Impact of IT-supported clinical pathways on medical staff satisfaction. A prospective longitudinal cohort study," International Journal of Medical Informatics, vol. 80, no. 3, pp. 151-156, 2011, doi: https://doi.org/10.1016/j.ijmedinf.2010.10.012.

[40] S. Barbagallo et al., "Optimization and planning of operating theatre activities: an original definition of pathways and process modeling.," BMC Medical Informatics \& Decision Making, vol. 15, no. 1, pp. 3853, 2015.

[41] K. H. Sung et al., "Application of clinical pathway using electronic medical record system in pediatric patients with supracondylar fracture of the humerus: a before and after comparative study.," BMC Medical Informatics \& Decision Making, vol. 13, no. 1, pp. 1-8, 2013.

[42] M.-M. Bouamrane and F. S. Mair, “A qualitative evaluation of general practitioners' views on protocoldriven eReferral in Scotland.," BMC Medical Informatics \& Decision Making, vol. 14, no. 1, pp. 1-24, 2014.

[43] M. J. Husain et al., "HERALD (health economics using routine anonymised linked data)," BMC medical informatics and decision making, vol. 12, no. 1, p. 24, 2012.

[44] N. Platt, M. Tarafdar, and R. Williams, "The complementary roles of Health Information Systems and Relational Coordination in alcohol care pathways: The case of a UK hospital," ECIS 2019 Proceedings, 2019.

[45] E. Øvrelid, T. Sanner, and A. Siebenherz, "Creating Coordinative Paths from admission to discharge: The role of lightweight IT in hospital digital process innovation," 2018.

[46] K. Eason and P. Waterson, "The implications of e-health system delivery strategies for integrated healthcare: Lessons from England," International Journal of Medical Informatics, vol. 82, no. 5, pp. e96e106, 2013, doi: https://doi.org/10.1016/j.ijmedinf.2012.11.004.

[47] M. Schriek, O. Türetken, and U. Kaymak, "A Maturity Model for Care pathways.," in Proceedings of ECIS 2016, 2016, p. ResearchPaper127, [Online]. Available: https://aisel.aisnet.org/cgi/viewcontent.cgi?article=1112\&context=ecis2016_rp.

[48] M. Juhrisch, G. Dietz, and H. Schlieter, "Towards Compliance in Organizational Engineering - A Case Study," in 20th Proceedings of European Conference on Informations System, Barcelona, 2012, p. Paper 168, [Online]. Available: https://aisel.aisnet.org/ecis2012/168/.

[49] M. Juhrisch, H. Schlieter, and G. Dietz, "Model-Supported Business Alignment of IT-Conceptual Foundations.," 2011, [Online].

Available: https://aisel.aisnet.org/cgi/viewcontent.cgi?article=1215\&context=amcis2011_submissions.

[50] J. Greenwood-Lee, G. Wild, and D. Marshall, "Improving accessibility through referral management: setting targets for specialist care," Health Systems, vol. 6, no. 2, pp. 161-170, Jul. 2017, doi: 10.1057/hs.2015.20.

[51] K. Baker et al., "Process mining routinely collected electronic health records to define real-life clinical pathways during chemotherapy," International Journal of Medical Informatics, vol. 103, pp. 32-41, 2017, doi: https://doi.org/10.1016/j.ijmedinf.2017.03.011.

[52] A. R. Cochran, K. M. Raub, K. J. Murphy, D. A. Iannitti, and D. Vrochides, "Novel use of REDCap to develop an advanced platform to display predictive analytics and track compliance with Enhanced Recovery After Surgery for pancreaticoduodenectomy," International Journal of Medical Informatics, vol. 119, pp. 54-60, 2018, doi: https://doi.org/10.1016/j.ijmedinf.2018.09.001.

[53] E. Aspland, D. Gartner, and P. Harper, "Clinical pathway modelling: a literature review," Health Systems, pp. 1-23, Sep. 2019, doi: 10.1080/20476965.2019.1652547.

[54] P. Richter, "Bringing Care Quality to Life: Towards Quality Indicator-Driven Pathway Modelling in Health Care Networks,” ECIS 2019 Proceedings, 2019.

[55] E. Demir, M. M. Gunal, and D. Southern, "Demand and capacity modelling for acute services using discrete event simulation," Health Systems, vol. 6, no. 1, pp. 33-40, Mar. 2017, doi: 10.1057/hs.2016.1.

[56] G. Berntsen, F. Strisland, K. Malm-Nicolaisen, B. Smaradottir, R. Fensli, and M. Røhne, "The Evidence Base for an Ideal Care Pathway for Frail Multimorbid Elderly: Combined Scoping and Systematic Intervention Review," J Med Internet Res, vol. 21, no. 4, p. e12517, Apr. 2019, doi: 10.2196/12517.

[57] C. Hufnagl, E. Doctor, L. Behrens, C. Buck, and T. Eymann, "Digitisation along the Patient Pathway in Hospitals," ECIS 2019 Proceedings, 2019.

[58] K. Flott, R. Callahan, A. Darzi, and E. Mayer, "A Patient-Centered Framework for Evaluating Digital Maturity of Health Services: A Systematic Review,” J Med Internet Res, vol. 18, no. 4, p. e75, Apr. 2016, doi: 10.2196/jmir.5047.

[59] F. Meng, C. K. Ooi, C. K. Keng Soh, K. Liang Teow, and P. Kannapiran, "Quantifying patient flow and utilization with patient flow pathway and diagnosis of an emergency department in Singapore," Health Systems, vol. 5, no. 2, pp. 140-148, Jun. 2016, doi: 10.1057/hs.2015.15.

[60] O. Korhonen and M. Isomursu, "Identifying Personalization in a Care Pathway: a Single-Case Study of a Finnish Healthcare Service Provider,” ECIS 2017 Proceedings, 2017. 